\title{
Role of Mitotic slippage in cancer resistance and DNA damage response in the MDA-MB-231-DOX-Treated Cells
}

\author{
Kristine Salmina *, Felikss Rumnieks, Ninel M Vainshelbaum, Dace Pjanova, Jekaterina Erenpreisa \\ Latvian Biomedical Research and Study Centre, LV-1067 Riga, Latvia
}

*kristine@biomed.lu.Iv

Introduction.

Mitotic slippage (MS) - mitosis failure and reversal to interphase with a doubled genome, is a typical response of TP53-mutant tumors resistant to genotoxic therapy. MS and associated micronucleation may play a role in escaping cell death via sorting of the intrinsically damaged DNA. The mechanisms of this MSaided cancer resistance are poorly understood.

Methods.

For experimental studies the metastatic triple-negative breast adenocarcinoma MDA-MB-231 cell line, was treated with $100 \mathrm{nM}$ DOX (doxorubicin) for $24 \mathrm{~h}$. After drug removal, cells were sampled over a 3-week period post-treatment until the appearance of escape clones.

Results.

After DOX treatment these cells polyploidize, display premature senescence, sort the damaged DNA into the cytoplasm and acquire an amoeboid phenotype and finally bud the depolyploidized progeny, restarting the mitotic cycling. We found selective release into the cytoplasm of telomere fragments enriched in telomerase reverse transcriptase (hTERT), telomere capping protein TRF2, and DNA double-strand breaks marked by $\mathrm{pH} 2 \mathrm{AX}$. This occurs along with the alternative lengthening of telomeres (ALT) and DNA repair by homologous recombination (HR) in the nuclear promyelocytic leukemia (PML) bodies, where we found colocalization of PML bodies with TRF2, RAD51 and meiotic nuclease SP011. The cells in repeated MS cycles activate meiotic genes (meiotic recombinase DMC1, meiotic cohesin REC8, meiotic kinase MOS) and display holocentric chromosomes characteristic for inverted meiosis (IM)

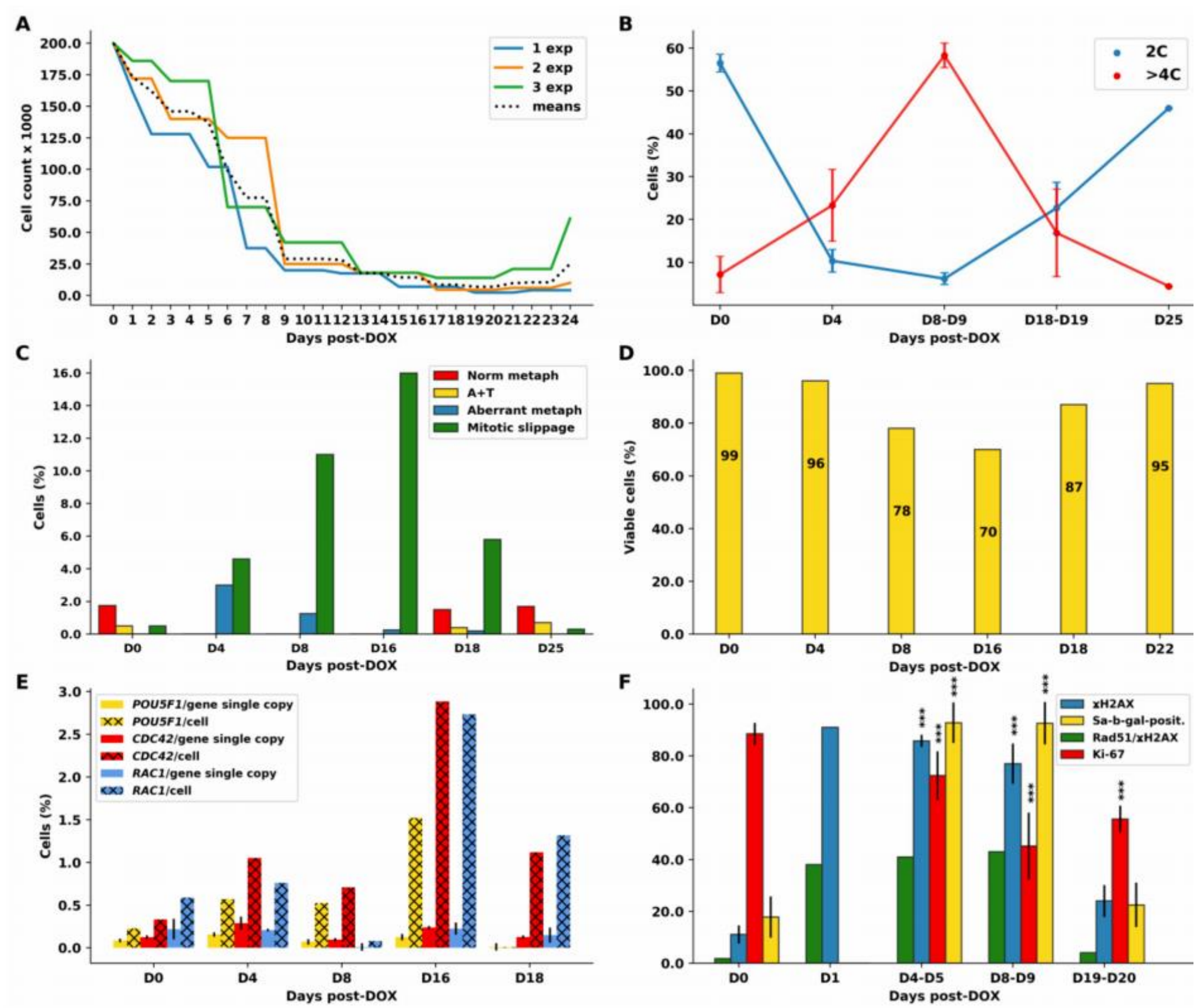

Fig.1. The quantified parameters of MDA-MB-231 cells following DOX treatment: $(A)$ the cell growth curves; (B) the reciprocal relationship between mitotic (2C) and polyploid $(>4 C)$ cell numbers; (C) representative differential mitotic counts; (D) viability test with trypan blue; (E) results of gene transcription evaluation obtained by Selfie digital PCR for three gene transcripts quantified per gene copy and per cell; and (F) the dynamics of the senescence marker Sa- $\beta$-gal and proliferation marker Ki-67 along with DNA doublestrand breaks $(\gamma \mathrm{H} 2 \mathrm{AX})$ and their repair by homologous recombination-cells with colocalized Rad51/ $/ \mathrm{H} 2 \mathrm{AX}$ foci. $p<0.001$

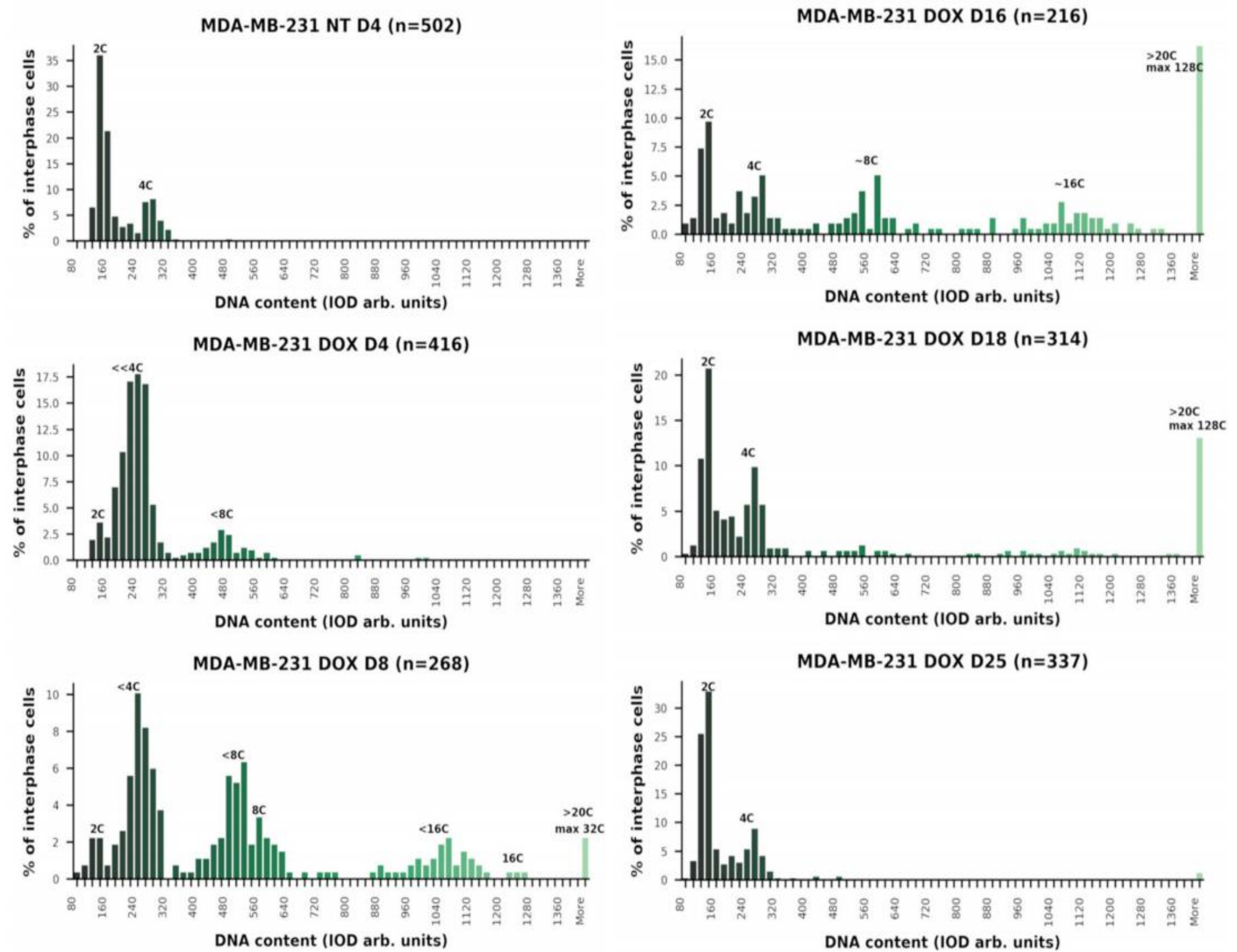

Fig.2. Cell cycle changes monitored with in situ DNA cytometry in the dynamics of the OX treatment experiment
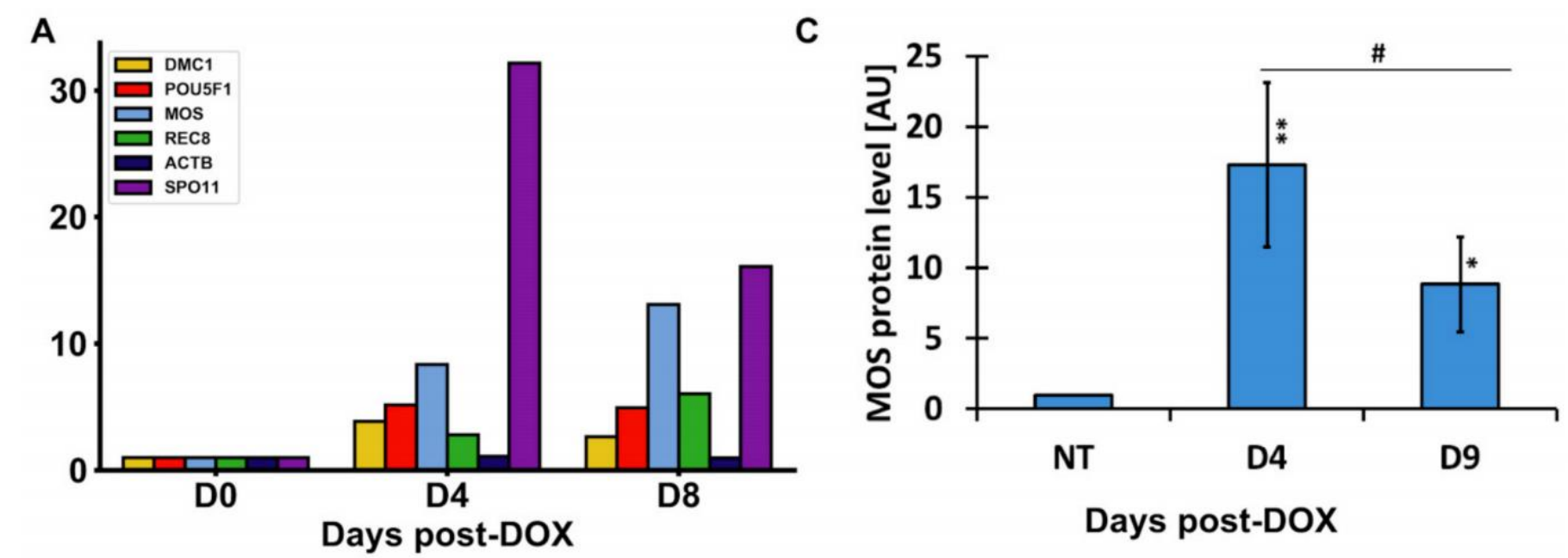

Fig.3. Expression of the meiotic genes and proteins after DOX treatment. (A) RT-PCR (C) Western Blot.
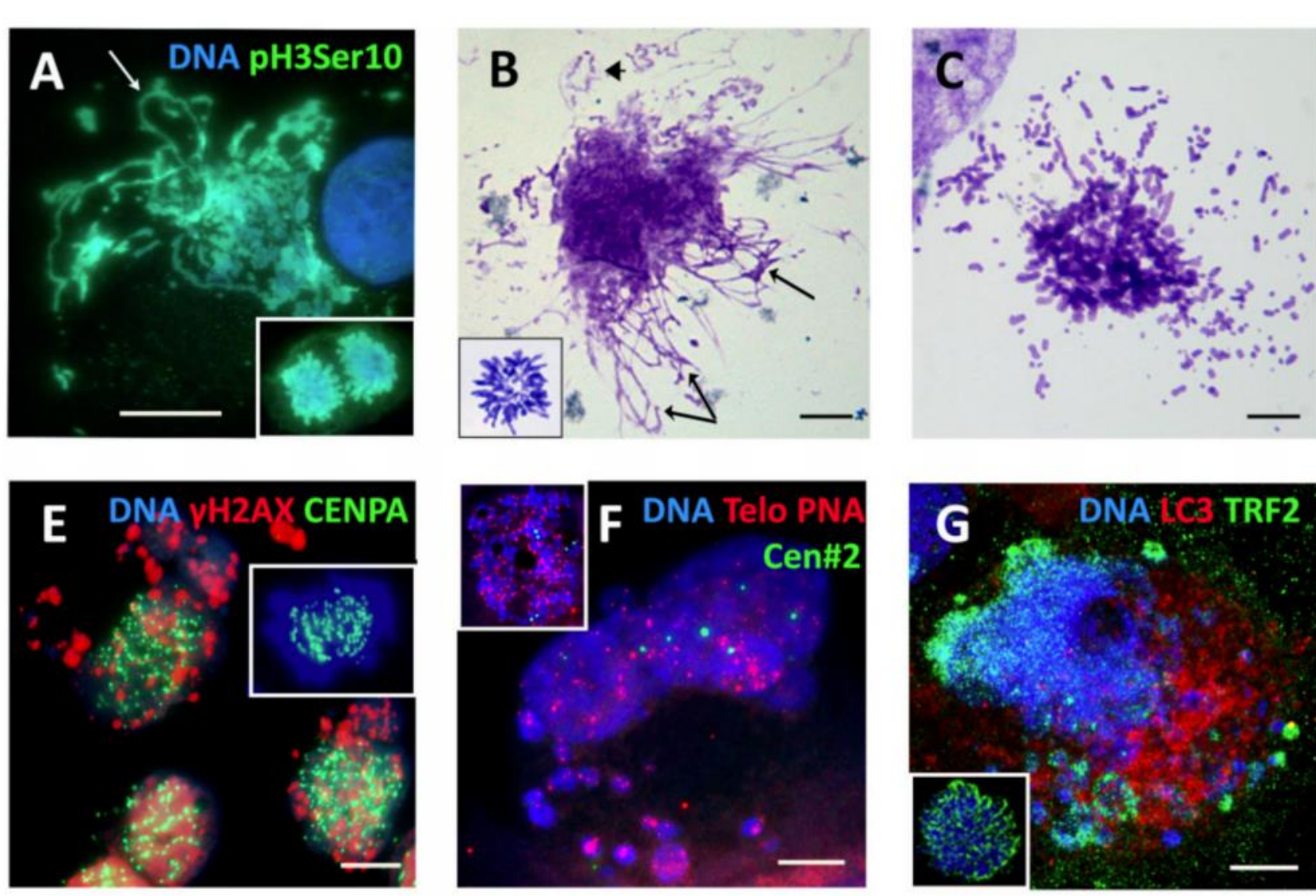

Fig.4. Aberrant mitosis and mitotic slippage (MS) post-DOX treatment. (A-C) Aberrant metaphases with loopy chromosomes. (A) DOX-D4. (B,C) DNA staining with Toluidine blue (DOX-D16 and D18). (E-G) Mitotic slippage: (E) holokinetic arrangement of kinetochores in the main nucleus and release of the damaged DNA into the cytoplasm (DOX-D5); (F) FISH with the telomere and cen\#2 probes showing retention of centromeres in the cell nucleus and release of a proportion of telomeres into the cytoplasm (DOX-D4); (G) preferential release of the TRF2-associated chromatin into the cytoplasm (DOX-D7);; (inserts: normal mitotic cells of NT).
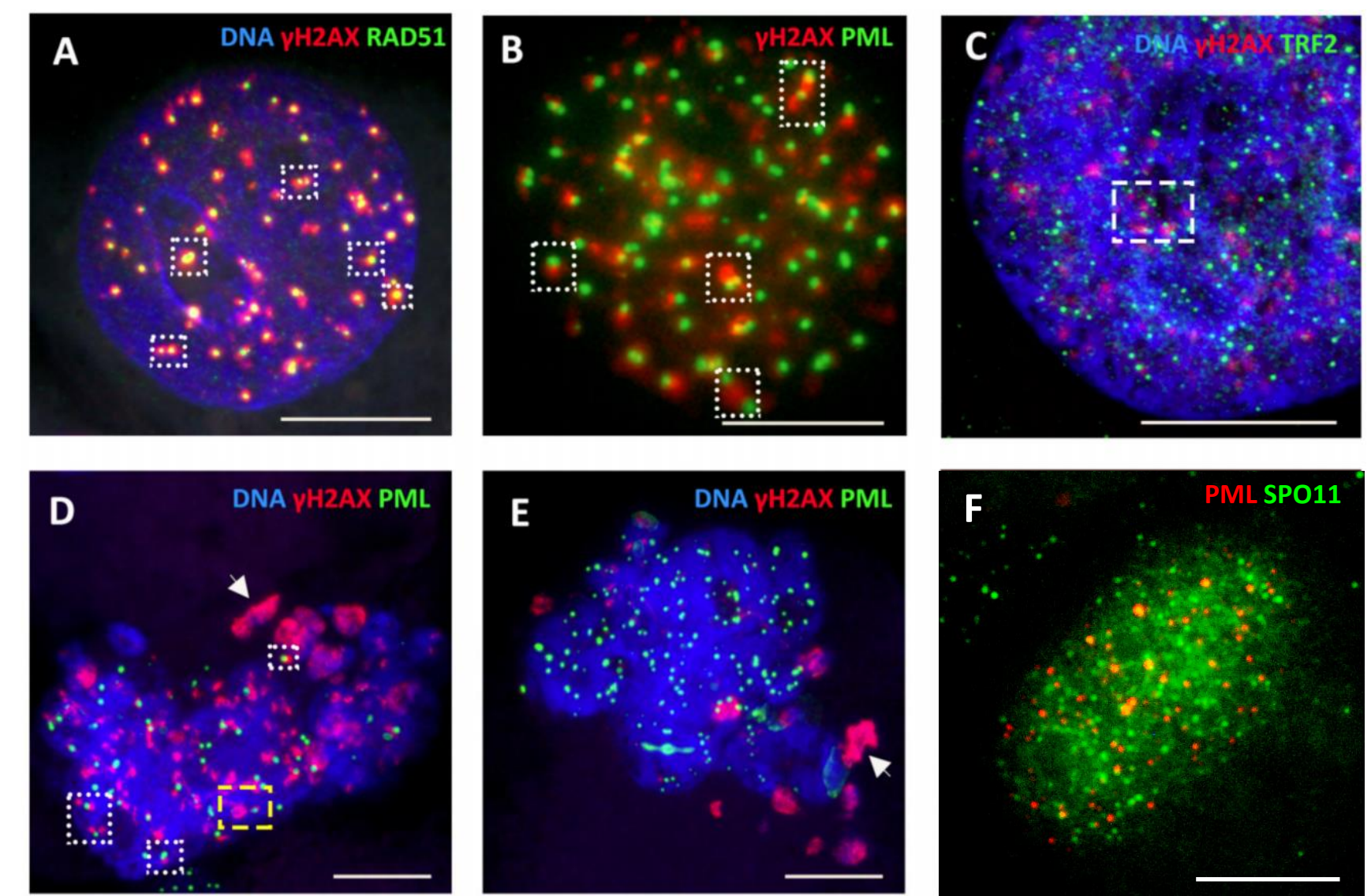

Fig.5. DNA repair of telomere DNA double-strand breaks by RAD51-dependent HR involving PML bodies, a sign of alternative lengthening of telomeres (ALT) in giant post-DOX cell nuclei undergoing MS cycles with sorting the damage signaling DNA out into the cytoplasm and reconstituting subnuclei free of it Days 5-9 post-DOX.
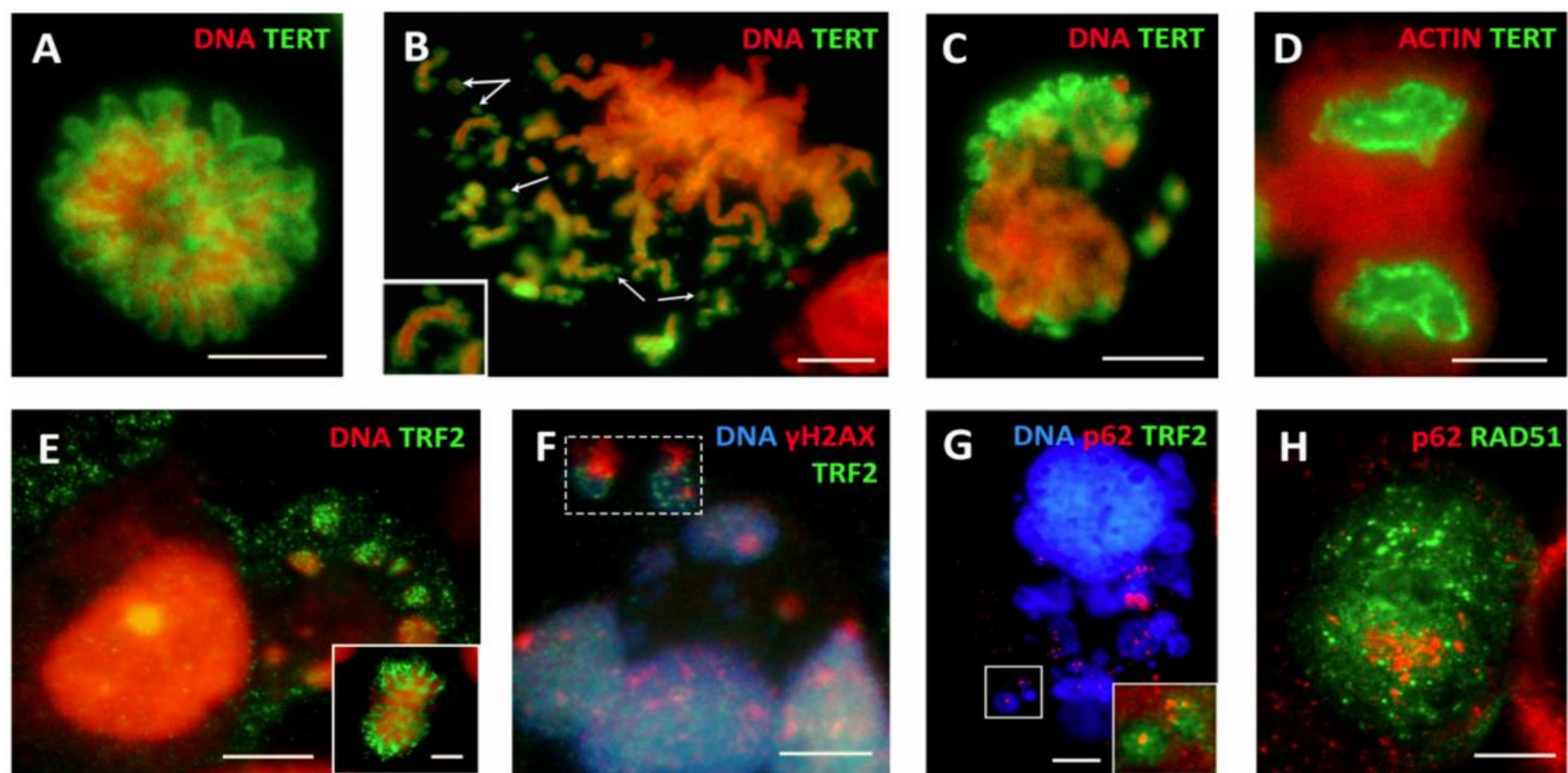

Fig.6. The release of the TERT-TRF2- $\mathrm{HH} 2 \mathrm{AX}$-marked chromatin into the cytoplasm of NT (A, insert E) and DOX-treated (B-H) cells accompanied by the autophagy adaptor SQSTM1/p62.

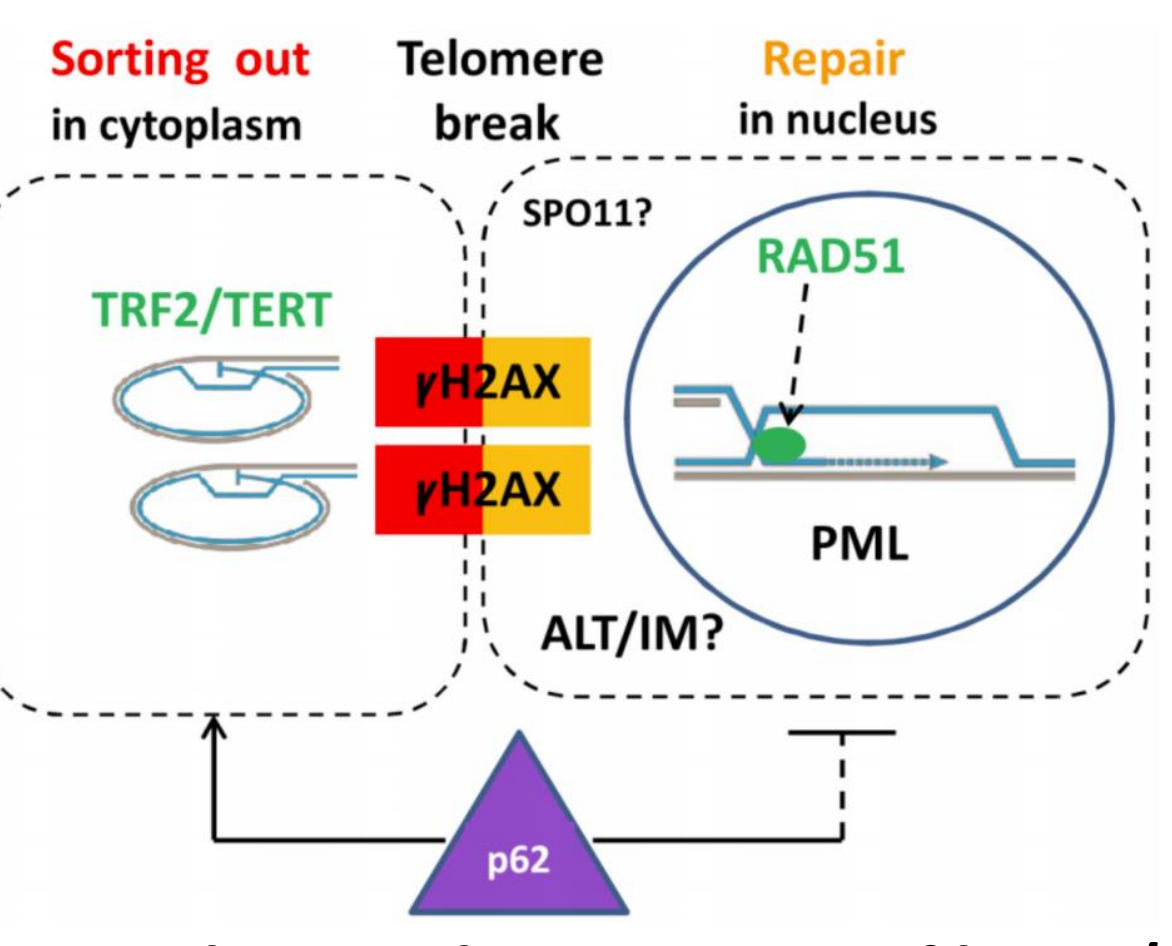

Fig.7. A schematic showing the cytoplasmic sorting of hTERT/TRF2-marked DNA damage signaling telomere ends cut off by a telomere break during mitotic slippage. This process is associated with the ALT-RAD51-driven repair by homologous recombination of the two co-aligned trimmed telomeres occurring in specific nuclear PML (APB) bodies. ALT may be coupled with inverted meiosis (IM) by recombining homologous chromosomes conjugated by telomeres at the same breakage site. In this case, the breakage sites can be introduced by meiotic nuclease SPO11. The ubiquitination protein SQSTM1/62 participates in the sorting of the extranuclear DNA.

Conclusion: Present results investigating the link between DNA damage response, cellular senescence, MS, and the processes of ALT and IM in chemoresistant cancer cells, all converging on telomeres, open a new avenue for further research and possible targeting.

Ref.: Salmina K., et al. "'Mitotic Slippage' and Extranuclear DNA in Cance Chemoresistance: A Focus on Telomeres." Int. J. Mol. Sci. 2020, 21, 2779.

Acknowledgements: This work was supported 